\title{
Influencia de la posición libremente escogida comparada con litotomía durante la atención de parto en los resultados maternos y neonatales
}

\author{
Influence of freely chosen position compared to lithotomy during \\ delivery care on maternal and neonatal outcomes
}

\author{
Sonia Sánchez Chávez, ${ }^{*}, \neq$ Roger Valdez Trujillo, ${ }^{*}$, José Alan Arvizu Armenta, ${ }^{\ddagger}$ \\ Pablo Mariano González Aldeco, ${ }^{\ddagger}$ Cecilio Rodríguez Ayala ${ }^{\ddagger}$ \\ Citar como: Sánchez CS, Valdez TR, Arvizu AJA, González APM, Rodríguez AC. Influencia de la posición \\ libremente escogida comparada con litotomía durante la atención de parto en los resultados maternos \\ y neonatales. Acta Med GA. 2022; 20 (1): 43-49. https://dx.doi.org/10.35366/103555
}

\section{Resumen}

Objetivo: Comparar resultados obstétricos y perinatales en partos atendidos en posición libremente escogida y litotomía. Material y métodos: Estudio descriptivo, retrospectivo y transversal. Se incluyeron embarazos únicos, edad gestacional $\geq 37$ semanas, presentación cefálica y partos eutócicos atendidos en un hospital de la Ciudad de México. Se analizaron datos demográficos, resultados obstétricos y neonatales. Se realizó un análisis univariado, utilizando $\chi^{2}$ para analizar variables cualitativas y $\mathrm{t}$ de Student o $\mathrm{U}$ de Mann-Whitney, según su distribución, para las variables cuantitativas. Resultados: Se incluyeron 100 pacientes con parto vaginal, divididas en dos grupos; el primero con 44 partos atendidos en posición libremente escogida comparados con 56 en posición de litotomía. Se encontró que en la posición libremente escogida ingresaron con mayor dilatación y borramiento cervical, requirieron menor analgesia obstétrica $(p<0.001)$, menos conducción del trabajo de parto con oxitocina $(p<0.001)$ y mejor puntuación de Apgar al minuto y 5 minutos $(p<0.001)$. No se observaron diferencias estadísticamente significativas en la duración del periodo expulsivo, desgarros perineales, ni pérdidas hemáticas $(p>$ $0.05)$. Conclusiones: La posición libremente escogida no tiene consecuencias adversas maternas ni neonatales. La posición de litotomía forzada está relacionada con intervenciones como uso de oxitocina y analgesia obstétrica.

Palabras clave: Resultados maternos, posición litotomía posición libremente escogida

\section{Abstract}

Objective: To compare obstetric and perinatal results in deliveries attended in freely chosen position and lithotomy. Material and methods: Descriptive, retrospective, and crosssectional study. Single pregnancies, gestational age $\geq 37$ weeks, cephalic presentation, and eutocic deliveries attended at a hospital in Mexico City were included. Demographic data, obstetric and neonatal outcomes were analyzed. A univariate analysis was performed, using $\chi^{2}$ to analyze qualitative variables and Student's t or Mann-Whitney $U$, according to their distribution, for quantitative variables. Results: 100 patients with vaginal delivery were included, divided into two groups; the first with 44 deliveries attended in a freely chosen position compared to 56 in the lithotomy position. It was found that in the freely chosen position, they were admitted with greater cervical dilation and effacement required less obstetric analgesia $(p<0.001)$, less conduction of labor with oxytocin ( $p$ $<0.001$ ), and better Apgar score at 1 minute and 5 minutes ( $p$ $<0.001$ ). No statistically significant differences were observed in the duration of the expulsive period, perineal tears, or blood loss $(p>0.05)$. Conclusions: The freely chosen position has no adverse maternal or neonatal consequences. The forced lithotomy position is related to interventions such as the use of oxytocin and obstetric analgesia.

Keywords: Maternal outcomes, lithotomy position, freely chosen position. * Facultad Mexicana de Medicina de la Universidad La Salle, México.
* Servicio de Ginecología y Obstetricia del Hospital Nuevo Sanatorio
Durango, Ciudad de México.
Correspondencia:

Dra. Sonia Sánchez Chávez

Correo electrónico: sonand.sanros@gmail.com

www.medigraphic.com/actamedica

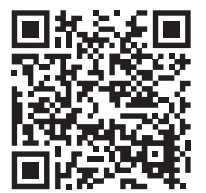




\section{INTRODUCCIÓN}

El parto es un evento que requiere la participación e interacción del pasaje, pasajero y la energía para conseguir su paso a través de la pelvis y el piso pélvico. Un componente importante es la pelvis, que se encuentra conformada por huesos, músculos, ligamentos y articulaciones que presentan cierta movilidad durante el embarazo, por efecto hormonal y cambios en posición también modifican sus diámetros.

El uso de las diversas posiciones para la atención obstétrica se ha modificado a lo largo del tiempo y continúa influida por aspectos socioculturales, Atwood las divide en dos: la vertical y la neutral. Las posiciones verticales que describe son de pie, sentado, en cuclillas y arrodillado; en estas posiciones, el peso recae principalmente sobre sus pies y las posiciones neutrales que incluye la semirreclinada, decúbito dorsal, litotomía y lateral con sus variantes. En dicha publicación se postula que la postura regula el efecto de la gravedad en el feto y la eficiencia muscular general en los esfuerzos expulsivos. ${ }^{2}$

Una revisión reciente evaluó los beneficios y riesgos de diversas posiciones durante la atención obstétrica y solamente recomienda las posiciones supina y litotomía en caso de preferencia de la paciente, los beneficios de las posiciones verticales y lateral se presentan en la Tabla $1 .^{3}$
Otras ventajas descritas son aumento de la capacidad respiratoria de la paciente, mayor apertura del canal vaginal y vulva, sinergia entre las contracciones uterinas con el esfuerzo del diafragma y músculos abdominales durante el pujo con menor uso de oxitocina, mejoría de los resultados ácido-base en los recién nacidos, disminuye posibilidad de prolapso del cordón, así como beneficios psicoafectivos. Como desventajas están el aumento en la pérdida sanguínea y trauma perineal con algunas posiciones. En cambio, las ventajas del uso de la posición horizontal son la comodidad del personal médico y favorecer la revisión médica. ${ }^{4}$

Un estudio Cochrane del 2017 sugiere varios beneficios de la postura vertical como reducción de la duración del segundo periodo del trabajo de parto con predominio en las primigestas, reducción de las tasas de episiotomía y en partos instrumentados, pero con mayor riesgo de desgarros de segundo grado y de hemorragias obstétricas. ${ }^{5}$ Un ensayo clínico realizado en población mexicana no encontró diferencias en cuanto a complicaciones obstétricas, resultados perinatales e intensidad del dolor; sin embargo, encontraron un acortamiento del segundo periodo del trabajo de parto. ${ }^{6}$

Según las recomendaciones de la Organización Mundial de la Salud del 2018, se debe animar la movilidad y la posición erguida durante el trabajo de parto, y durante el periodo expulsivo alentar a la paciente a adoptar la posición

Tabla 1: Beneficios de las diversas posiciones para la atención de parto.

\begin{tabular}{|c|c|c|}
\hline Aspecto evaluado & Posición & Mecanismo \\
\hline \multirow[t]{3}{*}{ Acelerar el trabajo de parto } & Vertical & Efectos de gravedad \\
\hline & Cuclillas & Contracciones más fuertes y efectivas* \\
\hline & Sentada & Aumento del diámetro pélvico \\
\hline \multirow[t]{6}{*}{ Alivio del dolor } & Vertical & Acorta el trabajo de parto \\
\hline & Cuclillas & \\
\hline & Semisentada & \\
\hline & Sentada & Evita que el peso recaiga en la cintura \\
\hline & & Mayor sensación de control y mejor autosuficiencia \\
\hline & & Acorta el trabajo de parto \\
\hline \multirow[t]{3}{*}{ Reducción del trauma perineal } & Lateral & \\
\hline & 4 puntos & Mejor visualización del periné y presión reducida sobre el periné \\
\hline & De rodillas & Permite libre movimiento y no hay presión externa sobre la pelvis \\
\hline \multirow{2}{*}{$\begin{array}{l}\text { Promover el bienestar fetal y del recién } \\
\text { nacido }\end{array}$} & Vertical & Evitan la compresión de los vasos intrabdominales \\
\hline & Lateral & \\
\hline \multirow{2}{*}{$\begin{array}{l}\text { Manejo de complicaciones obstétricas } \\
\text { (distocia de hombros, OPP) }\end{array}$} & 4 puntos & Desplazamiento del hombro impactado \\
\hline & L ateral & $\begin{array}{l}\text { Ampliar la salida pélvica } \\
\text { Meiora la rotación de la cabeza fetal }\end{array}$ \\
\hline
\end{tabular}

* Aún discutido el aumento de la intensidad de las contracciones. OPP = occipitoposterior persistente. 
que ella elija, incluso cuando tengan analgesia peridural. ${ }^{7}$ En cuanto a la normatividad en México, la guía de práctica clínica de nuestro país recomienda el empleo de la posición vertical con sus diferentes variedades en caso de contar con la infraestructura necesaria, y en el caso contrario, ofrecer alguna posición diferente a la litotomía, con la finalidad de estimular a las mujeres a tener su parto en la posición que les parezca más cómoda, inclusive cuando se utilice analgesia obstétrica. ${ }^{8}$ La Norma Oficial Mexicana-007, el artículo 2 de la Constitución Política de los Estados Unidos Mexicanos y la Ley General de Salud sugieren el uso de estas técnicas en la atención del parto, ya que consideran la posición vertical del parto como parte de los conocimientos y elementos que forman parte de la cultura indígena y presenta beneficios para la integridad perineal materna. ${ }^{9}$

Nuestro objetivo es evaluar si la atención de parto en posiciones diferentes a la litotomía presenta diferencias en su manejo, uso de analgesia, duración del periodo expulsivo, estado perineal y complicaciones postparto.

\section{MATERIAL Y MÉTODOS}

Estudio descriptivo, retrospectivo y transversal que incluyó pacientes cuyos partos fueron atendidos en un hospital de segundo nivel de la Ciudad de México, durante un periodo de 10 meses, de septiembre del 2017 a junio del 2018; elegidas de manera consecutiva. Los criterios de inclusión fueron: embarazos únicos, edad gestacional $\geq$ 37 semanas, presentación cefálica y partos eutócicos. Los criterios de exclusión fueron: edades gestacionales $<37$ semanas, presentaciones pélvicas, episiotomía durante la atención de parto, trabajos de parto cuya resolución fue vía abdominal y parto instrumentado. La muestra total fue de 100 pacientes, de las cuales, 44 fueron atendidas en posición libremente escogida (vertical con sus diferentes posiciones: sentada, de rodillas, en cuclillas, parada, así como semidecúbito, cuatro puntos y lateral) y el segundo conformado por 56 pacientes con partos atendidos en posición de litotomía.

Se elaboró una hoja de recolección con datos demográficos, obstétricos y resultados neonatales, y se revisaron los expedientes de las pacientes atendidas en un periodo de 10 meses. Dicha información se agrupó en una base de datos de Excel y se realizó análisis estadístico con el programa IBM SPSS Statistics versión 25.

Las variables maternas que se ocuparon fueron: edad, peso, talla, índice de masa corporal pregestacional, edad gestacional, paridad, aumento ponderal, dilatación y borramiento al ingreso, manejo del trabajo de parto, posición adoptada por la paciente durante la atención del parto, uso de analgesia obstétrica y complicaciones intra- o postparto; las variables neonatales evaluadas fueron: peso fetal, talla y Apgar; las variables perineales fueron presencia de desgarros, localización y grado de profundidad.

Para la edad materna y el índice de masa corporal, subdividimos en dos grupos para clasificar a las pacientes de riesgo, tanto por la edad como por la obesidad pregestacional.

Para las características cervicales tomamos dilatación cervical $>4 \mathrm{~cm}$ como trabajo de parto en fase activa y el borramiento fue expresado en porcentaje según el adelgazamiento del cuello.

La variable dependiente fue la posición durante el periodo expulsivo, éste se clasificó en posición libremente escogida y litotomía forzada; el parto en posición libremente escogida lo definimos como postura en la cual el peso de la paciente se distribuye sobre diversas partes del cuerpo como glúteos, pies o rodillas, excluyendo la espalda. Se incluyen la posición vertical con sus variantes, así como la posición de cuatro puntos y lateral; el parto en posición de litotomía forzada lo definimos como la postura en la cual la paciente descansa sobre su espalda y con las rodillas flexionadas y dirigidas hacia los lados y los talones en los estribos de la mesa de expulsión.

Análisis estadístico: Se realizó con el programa IBM SPSS Statistics versión 25. Las variables cualitativas se expresaron con número de eventos y porcentaje, las variables cuantitativas conforme a las pruebas de normalidad Kolmogórov-Smirnov, las variables con distribución normal se realizaron con media y desviación estándar, las variables con libre distribución se expresaron con mediana y rango intercuartílico. Se realizó un análisis univariado donde las variables cualitativas se analizaron con $\chi^{2}$ y las variables cuantitativas conforme a su distribución con t de Student en el caso de las variables con distribución normal y con $U$ de Mann-Whitney en el caso de variables con libre distribución.

\section{RESULTADOS}

Maternos y perineales: de las 100 pacientes incluidas en el estudio, hubo 44 en posición libremente escogida (LE) y 56 fueron atendidas en posición de litotomía forzada (LF). La media de la edad materna en el grupo de posición LE fue de $31.1 \pm 4.84$ años, comparado con $29.46 \pm 5.55$ en el grupo LF. La edad materna avanzada fue de $20.5 \%$ en el grupo LE y de $10.7 \%$ en el grupo LF. La edad gestacional tuvo una mediana de 39.4 (38.4-40.1) en LE y de 39.4 (38.3-40.0) en LF. La nuliparidad estuvo presente en $61.4 \%$ de las mujeres de LE y en $35.7 \%$ de las pacientes del grupo LF. Un resumen de las características generales de las pacientes se presenta en la Tabla 2.

Respecto al aumento ponderal, se observó una media de $10.66 \pm 3.39 \mathrm{~kg}$ en el grupo LE y una media de 10.92 
Tabla 2: Características demográficas maternas en cada posición durante el periodo expulsivo (N = 100).

\begin{tabular}{lcc}
\hline & $\begin{array}{c}\text { Posición libremente escogida }(\mathrm{N}=44) \\
n(\%)\end{array}$ & $\begin{array}{c}\text { Litotomía forzada (N = 56) } \\
n(\%)\end{array}$ \\
Variable & & $50(89.3)$ \\
\hline Edad (años) materna & $35(79.5)$ & $6(10.7)$ \\
$\quad<36$ & $9(20.5)$ & $74.64 \pm 12.31$ \\
$\geq 36$ & $72.76 \pm 11.11$ & $1.59 \pm 0.05$ \\
Peso $(\mathrm{kg})^{*}$ & $1.61 \pm 0.06$ & $36(64.3)$ \\
Talla $(\mathrm{m})^{*}$ & & $20(35.7)$ \\
IMC $\left(\mathrm{kg} / \mathrm{m}^{2}\right)$ & $31(70.5)$ & $39.4(38.3-40.0)$ \\
$\quad<30$ & $13(29.5)$ & $36(64.3)$ \\
$\geq 30$ & $39.4(38.4-40.1)$ & $20(35.7)$
\end{tabular}

$\pm 4.60 \mathrm{~kg}$ en el grupo LF. El valor de $p$ fue de 0.75 y en comorbilidades fue de 0.34 .

Las características cervicales al momento del ingreso se presentan en la Tabla 3. En lo referente al manejo durante el trabajo de parto, en el grupo LE, 33 pacientes (75\%) se mantuvieron a libre evolución, ocho (18.2\%) tuvieron conducción y tres (6.8\%) fueron inducciones; en el grupo LF, 36 (64.3\%) pacientes estuvieron en conducción, 19 (33.9\%) fueron libre evolución y una (1.8\%) fue inducción del trabajo de parto. Al comparar el uso de analgesia obstétrica en ambos grupos se encontró que 4.5\% la solicitó en el grupo LE, comparado con $58.9 \%$ en el grupo LF. Al analizar con $\chi^{2}$ el manejo durante el trabajo de parto y analgesia obstétrica, la p fue $<0.001$ (Tabla 3).

En cuanto a la duración del periodo expulsivo, la media en el grupo LE fue de $62.7 \pm 11.03$ minutos y de $63 \pm 21.8$ minutos en LF, el tiempo máximo observado en este último grupo fue de 180 minutos. Al comparar las medias de las variables con la prueba de $t$ se obtuvo un valor de p de 0.73 (Tabla 3).

En lo concerniente a la evaluación perineal, se analizaron presencia de desgarros, localización y grado de desgarro en caso de que se situaran en región perineal. Al comparar los sitios de localización de los desgarros, encontramos un total de 102, dado que algunas pacientes presentaron más de un desgarro; el grupo LE tuvo 44 desgarros y el $L F$, 58. No se encontraron desgarros vaginales en LE; sin embargo, en LF se presentaron en $8.6 \%$ de las pacientes. En lo referente a los desgarros que se presentaron en más de una localización, en el grupo de LE se encontraron seis pacientes $(13.6 \%)$, comparado con LF que tuvieron
11 pacientes (19.6\%); en ambos grupos, la combinación de perineal con parauretral fue predominante. En el caso de los desgarros perineales también se valoró el grado de profundidad según la clasificación de Sultan (Tabla 3).

Resultados neonatales: al comparar el peso al nacer observamos que la media en el grupo de LE fue de 3,132.1 \pm 383.33 gramos vs $3,076.61 \pm 371.5$ gramos en el grupo de LF. En cuanto al Capurro, la mediana fue de 39.3 (3940) semanas en el grupo LE y de 39.2 (38.5-40) semanas en LF. En ambas variables no se encontraron diferencias estadísticamente significativas con p de 0.46 y 0.63 , respectivamente. La valoración de Apgar al minuto y 5 minutos presenta significancia estadística (Tabla 4).

\section{DISCUSIÓN}

La atención del parto ha sufrido cambios a lo largo del tiempo y está influida muchas veces por aspectos socioculturales. En nuestro país continúa usándose ampliamente la posición de litotomía para la atención obstétrica, a pesar de las recomendaciones de organismos como la OMS.

Un metaanálisis Cochrane del $2017^{5}$ menciona algunas ventajas y desventajas de las posiciones verticales sobre las supinas; como ventajas están la reducción en la duración del periodo expulsivo, sobre todo en primigestas, menores tasas de episiotomía y partos instrumentados; las desventajas que encontraron fueron mayor riesgo de pérdidas sanguíneas, superiores a $500 \mathrm{~mL}$, y más riesgo de desgarros perineales de segundo grado. En contraste, en nuestro estudio no encontramos mayor riesgo de hemorragia obstétrica, 
Tabla 3: Características obstétricas.

\begin{tabular}{|c|c|c|c|}
\hline Variable & $\begin{array}{l}\text { Posición libremente escogida } \\
\qquad(\mathrm{N}=44)\end{array}$ & $\begin{array}{l}\text { Litotomía forzada } \\
\qquad(\mathrm{N}=56)\end{array}$ & $n$ \\
\hline varlable & & & $p$ \\
\hline Aumento ponderal $(\mathrm{kg})^{\ddagger}$ & $10.66 \pm 3.39$ & $10.92 \pm 4.60$ & $0.75^{\star}$ \\
\hline Dilatación cervical al ingreso (cm) & & & $0.005^{\star \star}$ \\
\hline$<4$ & $10(22.7)$ & $27(48.2)$ & \\
\hline$\geq 4$ & $34(77.3)$ & $29(51.8)$ & \\
\hline Borramiento cervical al ingreso $(\%)^{\S}$ & $80(60-90)$ & $50(40-80)$ & $<0.001^{\star *}$ \\
\hline Manejo del trabajo de parto & & & $<0.001^{* * *}$ \\
\hline Inducción & $3(6.8)$ & $1(1.8)$ & \\
\hline Conducción & 8 (18.2) & $36(64.3)$ & \\
\hline Libre evolución & $33(75.0)$ & 19 (33.9) & \\
\hline Analgesia obstétrica & $2(4.5)$ & $33(58.9)$ & $<0.001^{\star * *}$ \\
\hline Duración del periodo expulsivo $(\min )^{\ddagger}$ & $62.7 \pm 11.03$ & $63 \pm 21.8$ & $0.73^{\star}$ \\
\hline Evaluación perineal $\|^{\pi}$ & $38(86.4)$ & $47(83.9)$ & $0.48^{\star \star \star}$ \\
\hline Presencia de desgarros & $34(77.3)$ & $37(63.8)$ & $0.158^{\star \star \star}$ \\
\hline \multicolumn{4}{|l|}{ Perineal } \\
\hline $1^{0}$ & $17(50.0)$ & $21(56.8)$ & $0.165^{\star \star *}$ \\
\hline $2^{0}$ & $17(50.0)$ & $13(35.1)$ & \\
\hline $3^{\circ} \mathrm{A}$ & $0(0.0)$ & $3(8.1)$ & \\
\hline Parauretral & $6(13.6)$ & $10(17.2)$ & $0.386^{\star \star *}$ \\
\hline Vaginal & $0(0.0)$ & $5(8.6)$ & $0.051^{\star \star \star}$ \\
\hline Labios menores & $4(9.1)$ & $6(10.4)$ & $0.531^{\star \star \star}$ \\
\hline
\end{tabular}

\section{Tabla 4: Resultados neonatales.}

\begin{tabular}{|c|c|c|c|}
\hline Variable & $\begin{array}{l}\text { Posición libremente escogida } \\
\qquad \begin{array}{c}(\mathrm{N}=44) \\
\mathrm{n}(\%)\end{array}\end{array}$ & $\begin{array}{l}\text { Litotomía forzada } \\
\qquad \begin{array}{c}\mathrm{N}=56) \\
\mathrm{n}(\%)\end{array}\end{array}$ & $\mathrm{p}$ \\
\hline Peso $(g)^{\ddagger}$ & $3,132.1 \pm 383.33$ & $3,076.61 \pm 371.5$ & $0.46^{*}$ \\
\hline Talla $(\mathrm{cm})^{\S}$ & $50(49-51.7)$ & $50(49.1-51)$ & $0.54^{\star \star}$ \\
\hline Capurro & $39.3(39-40)$ & $39.2(38.5-40)$ & $0.63^{\star *}$ \\
\hline Apgar al minuto & & & $<0.001^{\star * *}$ \\
\hline 7 & $0(0.0)$ & $2(3.6)$ & \\
\hline 8 & 5 (11.4) & $25(44.6)$ & \\
\hline 9 & $39(88.6)$ & $29(51.8)$ & \\
\hline Apgar a los 5 minutos & & & $<0.001^{* * *}$ \\
\hline 9 & $34(77.3)$ & 55 (98.2) & \\
\hline 10 & $10(22.7)$ & $1(1.8)$ & \\
\hline
\end{tabular}


ni diferencias significativas en la presencia de desgarros ( $p$ $=0.48)$ ni en la duración del periodo expulsivo $(p=0.73)$.

El estudio de Moraloglu ${ }^{10}$ encontró menor duración del periodo expulsivo, menor requerimiento de oxitocina, menor puntaje de dolor; sin diferencias en resultados neonatales. Nuestro estudio mostró que la posición libremente escogida tuvo una marcada reducción del requerimiento de analgesia obstétrica ( $p<0.001)$, así como menor conducción del trabajo de parto $(p<0.001)$. El menor uso de analgesia podría ser causado por el libre movimiento y deambulación durante el trabajo de parto, lo que está descrito en varias publicaciones como método no farmacológico para el control del dolor obstétrico.

En China se realizó un ensayo clínico aleatorizado ${ }^{11}$ que comparó la posición de cuatro puntos con la supina e informó tasas más altas de periné intacto, menores tasas de episiotomía, mayor duración del expulsivo y sin diferencias en el sangrado postparto ni en la puntuación de Apgar al minuto y los 5 minutos en la posición de cuatro puntos. Contrariamente a este estudio, el nuestro mostró que la posición libremente escogida está relacionada con mejor puntuación de Apgar con diferencias estadísticamente significativas ( $p<0.001$ ). Esto podría estar relacionado con la menor compresión de los vasos intraabdominales y tasas ligeramente más altas de lesiones perineales de primer y segundo grado; sin embargo, no difieren significativamente.

A nivel nacional, un estudio ${ }^{6}$ realizado en el 2013 que comparó a pacientes en posición supina y en posición vertical, no encontró diferencias entre las complicaciones obstétricas, intensidad del dolor, ni en resultados neonatales; no obstante, reporta acortamiento del segundo periodo y mayores desgarros vaginales en posición vertical. Estos resultados contrastan con los nuestros, ya que en la posición libremente escogida no encontramos desgarros vaginales, además de observar menor solicitud de analgesia obstétrica ( $p<0.001$ ); esto podría ser causado por una mayor apertura del canal vaginal al adoptar cambios en la posición materna.

El grupo con posición libremente escogida ingresó en su mayoría con trabajo de parto en fase activa, lo cual puede estar relacionado con la educación prenatal y los cursos psicoprofilácticos en los que se instruye a las embarazadas acerca del manejo no farmacológico de dolor y fisiología del trabajo de parto, lo que conlleva a un ingreso hospitalario en fases más avanzadas del trabajo de parto; sin embargo, para este estudio no se investigó si habían llevado el curso o no.

La fortaleza de nuestro estudio radica en que existen pocos estudios en nuestro país que evalúen las diversas posiciones para la atención del parto y sus resultados maternos y neonatales, ya que no es una práctica que se realice ni se difunda.
No obstante, tiene varias limitaciones, como el pequeño tamaño de la muestra, por lo que una muestra más grande podría arrojar mayores resultados; es un estudio retrospectivo donde sólo podemos analizar los resultados obtenidos y no se puede aleatorizar la distribución de los pacientes. Finalmente, en nuestro estudio no fue posible la subclasificación de todas las posiciones adoptadas por las pacientes que optaron por la libre posición, ya que al tratarse de un estudio retrospectivo dependemos de la información plasmada en el expediente; pero consideramos que un estudio que las divida aportaría más información.

\section{CONCLUSIONES}

La posición libremente escogida no tiene consecuencias nocivas maternas ni neonatales. Este estudio demuestra que la posición de litotomía forzada está relacionada con intervenciones como el uso de oxitocina para el manejo de trabajo de parto y analgesia obstétrica.

\section{REFERENCIAS}

1. Cunningham FG, Leveno KJ, Bloom S, Hauth JC, Rouse DJ, Spong CY. Williams obstetricia. 23a ed. México: McGraw-Hill; 2011. pp. 29-31.

2. Atwood RJ. Parturitional posture and related birth behavior. Acta Obstet Gynecol Scand Suppl. 1976; 57: 1-25.

3. Huang J, Zang Y, Ren LH, Li FJ, Lu H. A review and comparison of common maternal positions during the second-stage of labor. Int J Nurs Sci. 2019; 6 (4): 460-467. doi: 10.1016/j.ijnss.2019.06.007.

4. Dirección General de Planeación y Desarrollo en Salud. Modelo de atención a las mujeres durante el embarazo, parto y puerperio. Enfoque humanizado, intercultural y seguro. Manual de procesos y actividades para fortalecer la sensibilización y capacitación del personal de salud, y su implantación en el Sistema Nacional de Salud. México: 2015 [Consultado en septiembre de 2020]. Disponible en: https://www.gob.mx/cms/uploads/attachment/file/29343/ GuialmplantacionModeloParto.pdf

5. Gupta JK, Sood A, Hofmeyr GJ, Vogel JP. Position in the second stage of labour for women without epidural anaesthesia. Cochrane Database Syst Rev. 2017; 5 (5): CD002006. doi: 10.1002/14651858. CD002006.pub4.

6. Calvo AO, Flores RAL, Morales GVE. Comparison of obstetric and perinatal results of childbirth vertical position vs childbirth supine position. Ginecol Obstet Mex. 2013; 81 (01): 1-10.

7. WHO recommendations: intrapartum care for a positive childbirth experience. Geneva: World Health Organization; 2018 [Access September 2020]. Available in: https://www.who.int/ reproductivehealth/publications/intrapartum-care-guidelines/en/

8. Vigilancia y atención amigable en el trabajo de parto en embarazo de bajo riesgo. Guía de práctica clínica: guía de evidencias y recomendaciones. México: CENETEC; 2019 [Consultado en agosto de 2020]. Disponible en: http://imss.gob.mx/profesionales-salud/ gpc

9. Norma Oficial Mexicana NOM-007-SSA2-2016, Para la atención de la mujer durante el embarazo, parto y puerperio, y de la persona recién nacida. [Consultado en septiembre de 2020]. Disponible 
en: https://www.cndh.org.mx/sites/default/files/doc/Programas/VIH/ Leyes\%20y\%20normas\%20y\%20reglamentos/Norma\%20Oficial\%20 Mexicana/NOM-007-SSA2-2016\%20Embarazo\%2C\%20parto\%20 y\%20puerperio.pdf

10. Moraloglu O, Kansu-Celik H, Tasci Y, Karakaya BK, Yilmaz Y, Cakir $E$ et al. The influence of different maternal pushing positions on birth outcomes at the second stage of labor in nulliparous women. J Matern Fetal Neonatal Med. 2017; 30 (2): 245-249. doi: 10.3109/14767058.2016.1169525.
11. Zhang H, Huang S, Guo X, Zhao N, Lu Y, Chen M et al. A randomised controlled trial in comparing maternal and neonatal outcomes between hands-and-knees delivery position and supine position in China. Midwifery. 2017; 50: 117-124. doi: 10.1016/j.midw.2017.03.022.

Conflicto de intereses: Los autores declaran que no tienen ningún conflicto de intereses. 